\title{
NEW COMPENSATION SCHEME OF MAGNETO-OPTICAL CURRENT SENSOR FOR TEMPERATURE STABILITY IMPROVEMENT
}

\author{
Jinling Chen ${ }^{1)}$, Hongbin $\mathrm{Li}^{1)}$, Mingming Zhang ${ }^{1)}$, Yan Zhang ${ }^{2)}$ \\ 1) Huahzong University of Science and Technology, Rd-Luoyu 1037, Wuhan, Hubei, 430074, China(هjlfocus@163.com) \\ 2) Liaoning University of Technology, St-Shiying 169, D-Guta, Jinzhou, Liaoning, 121001, China
}

\begin{abstract}
A novel magneto-optical current sensor (MOCS) with two sensing arms is proposed to improve the temperature stability. One of the arms, with a highly stable permanent magnet attached and orthogonal to the other one, is designed to provide a reference that follows the temperature characteristics of the sensing material. By a normalization operation between two arms, the temperature drift is compensated adaptively and a sensing output proportional to the measured current can be reached. A dual-input and dual-output structure is specially designed for the reference sensing arm to demodulate the DC Faraday rotation angle. This scheme compensates simultaneously two main temperature influence factors, the Verdet constant and linear birefringence. Validation tests were carried out and are discussed.
\end{abstract}

Keywords: magneto-optical current sensor, normalization, Verdet constant, birefringence.

(C) 2012 Polish Academy of Sciences. All rights reserved

\section{Introduction}

The application of Magneto-optical Current Sensors (MOCS) has become an important branch of current detection in recent decades. In high voltage applications in a power electric system, MOCS was thought by researchers and electrical manufactures an ideal scheme for current transducing because of its inherent electrical insulation, immunity to electromagnetic interference, and tending smaller size and weight. Most power stations select current transformers with an iron core rather than MOCS because the MOCS is sensitive to temperature and the current measurement precision is limited in an outdoor environment. Temperature dependence baffles the population of MOCS in power system or some other areas.

MOCS employs the Faraday effect of magneto-optical material, such as diamagnetic flint glass or paramagnetic rare-earth glass, as sensing principle. Two critical factors that result in the temperature variation of MOCS are internal linear birefringence and Verdet constant of the sensing material [1]. Many compensation schemes have been designed to achieve excellent temperature stability [2-6] and most of them aim at weakening one of the effect factors instead of both. However, the MOCS accuracy must be better than $0.5 \%$ or even $0.2 \%$ in a wide operating temperature range from $-25^{\circ} \mathrm{C}$ to $40^{\circ} \mathrm{C}$ [7], since it works in an outdoor power station and for measurement or protection purpose.

A newly designed MOCS which combines optical and comparative measurement is proposed. It introduces an extra reference sensing arm with a permanent magnet perpendicular to the ordinary one. Both arms made from the same material are of identical size and shape. By comparing the ordinary Faraday rotation with the reference one, similarly to a normalization operation, the temperature stability of MOCS is improved. This scheme 
compensates both of above mentioned two factors simultaneously and deftly without any temperature monitoring unit or further research of material performance.

\section{Temperature characteristics of an ordinary MOCS}

The Faraday Magneto-optic effect can be described as a rotation of the plane of polarization which occurs when linearly-polarized light propagates through a magneto-optic material in the presence of a magnetic field, as shown in Fig. 1.

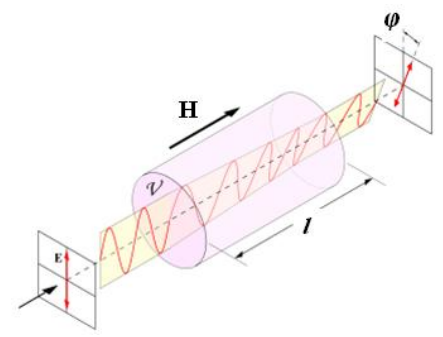

Fig. 1. Faraday magneto-optic effect.

The rotation angle $\varphi_{0}$ of a MOCS can be described as:

$$
\varphi_{0}=V H l,
$$

where $l$ is the active length of light path in the sensor, $H$ is the average magnetic field intensity on the active light path, $V$ is the Verdet constant of sensing material. Obviously, the rotation is proportional to the magnetic field which is proportional to the measured current in an ideal condition. The current value can be reached by detecting the rotation angle. However, residual stress in process exits in the magneto-optic material which causes linear birefringence $\delta$ and the Faraday rotation attenuation $k_{\delta}$, then the Faraday rotation changes to

$$
\varphi=k_{\delta} V H l
$$

$V$ varies with temperature. Reference [8] tells the temperature coefficient of the Verdet constant for diamagnetic material such as flint glass is about $10^{-4} /{ }^{\circ} \mathrm{C}$. When the temperature change is up to $65^{\circ} \mathrm{C}$, the MOCS sensitivity variation is about $0.65 \%$, beyond the error limit $0.5 \%$ or $0.2 \%$. The Verdet constant variation of paramagnetic material such as rare-earth glass is even much more severe. The effect of linear birefringence $k_{\delta}$ is relatively complicated. It changes not only with temperature $T$ but also with its gradient $ə T / \ni t$. Considering the temperature effect, the Faraday rotation angle $\varphi$ can be described as:

$$
\varphi(T)=V(T) H l k_{\delta}(T, \partial T / \partial t) .
$$

The exact expression of $\varphi(T)$ is hardly found out. And also it is difficult to achieve excellent improvement by the way of monitoring the sensor temperature and compensating in software since the temperature gradients are different in different regions and seasons.

\section{Newly designed MOCS}

\subsection{Principle}

The newly-designed MOCS has a new structure, as shown in Fig. 2. It includes two sensing arms made of magneto-optical glass and a newly- introduced highly stable permanent magnet, 
such as a samarium cobalt one, which generates a permanent magnetic field as reference. Arm 1 senses the measured current magnetic field $H_{x}$ and arm 2 senses the reference one $H_{N}$. These two magnetic fields are orthogonal to each other to avoid a mutual effect so the two arms are fixed perpendicularly in a box. Both pieces of arms are obtained from the same homogeneous bulk of glass and have the same sizes and shapes.

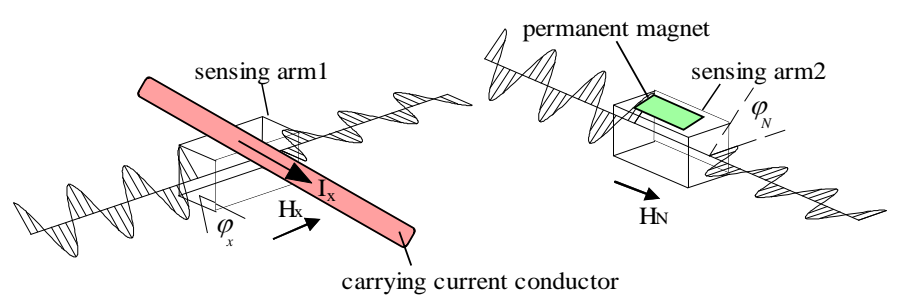

Fig. 2. Structure of comparative MOCS.

The Faraday rotation angles in two arms are respectively described as:

$$
\varphi_{x}=k_{\delta x} V_{x} H_{x} l, \varphi_{N}=k_{\delta N} V_{N} H_{N} l .
$$

$H_{x}, H_{N}$ are the average magnetic field intensities along the light path of arm 1 and 2, and obviously $H_{x}$ is proportional to the current $I_{x} . k_{\delta x}, k_{\delta N}$ are respectively the Faraday rotation attenuation caused by internal linear birefringence of two arms.

Put two arms in a box with a heat isolation coat to assure they are in the same temperature field. The temperature and its gradient values inside them are almost equal and then the effect of linear birefringence and Verdet constant changes in them can be assumed equal:

$$
k_{\delta x}=k_{\delta N}=k_{\delta 0}, V_{x}=V_{N}=V_{0} .
$$

The compensation is implemented by comparing two Faraday rotation angles, similarly to normalization:

$$
S=\frac{\varphi_{x}}{\varphi_{N}}=\frac{k_{\delta x} V_{x} H_{x} l}{k_{\delta N} V_{N} H_{N} l}=\frac{k_{\delta 0} V_{0} H_{x}}{k_{\delta 0} V_{0} H_{N}}=\frac{H_{x}}{H_{N}}=k I_{x} .
$$

The angle $\varphi_{N}$ follows the temperature characteristics of the sensing material, the normalization makes that $S$ is only proportional to the primary current, irrelevant to the Verdet constant and internal linear birefringence of the sensor.

\subsection{Demodulation}

The angles $\varphi_{x}$ and $\varphi_{N}$ have to be demodulated for comparison operation. The conventional demodulating method is to set a magneto-optical polarizer at the input end of the sensing arm and a magneto-optical analyzer at the output end, with $45^{\circ}$ included angle between two polarizing axes. Then according to Malus law [9] the sensor's output intensity $I_{o}$ is:

$$
I_{o}=\frac{I_{i}}{2} \cos ^{2}\left(45^{\circ}+\varphi\right)=\frac{I_{i}}{2}(1+\sin 2 \varphi) \approx \frac{I_{i}}{2}(1+2 \varphi)=\frac{I_{i}}{2}+I_{i} \varphi .
$$

After convert of the magneto-optical signal $I_{o}$ to an proportional electric signal by an O/E converter, the basic light intensity $I_{i} / 2$, a DC component, and the Faraday rotation proportional part $I_{i} \varphi$, an AC component, can be split by a properly designed filter, then the Faraday rotation angle $\varphi$ can be obtained by dividing the split AC component by the $\mathrm{DC}$ one, as given below, 


$$
S=I_{i} \varphi /\left(I_{i} / 2\right)=2 \varphi .
$$

It must be pointed out that this demodulating method is only suitable for alternating $\varphi$. When $\varphi$ is a DC quantity, it's impossible to split two components of Eq. 7 by a filter since both of them are in the same frequency domain.

The angle $\varphi_{x}$ is proportional to the primary current whose frequency is $50 \mathrm{~Hz}$ or $60 \mathrm{~Hz}$. It can be demodulated in this way. That means arm1 needs only one polarizer, one analyzer and one light source. $\varphi_{N}$ is a DC component since it is proportional to the magnetic density which is generated by the samarium cobalt magnet and permanent. It cannot be demodulated by the above way. A novel demodulating scheme, called dual-input and dual-output sensor, is designed to solve the problem, as shown in Fig. 3.

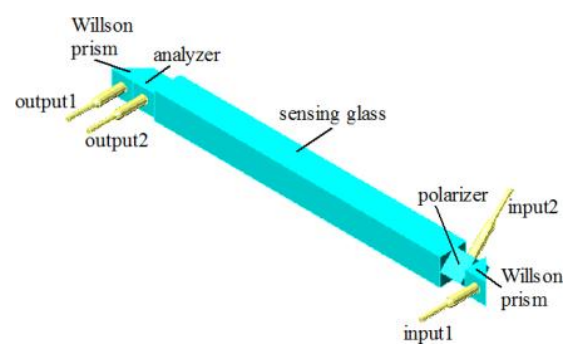

Fig. 3. Dual-input and dual-output sensor.

The sensor needs two light sources which are lighted alternately. The polarizing axis of the analyzer is set at an angle of $45^{\circ}$ to that of the polarizer. By means of two Willson prisms to change the propagating direction of light, the structure is effectively two polarizer-analyzer pairs, as shown in Fig. 4, and the axis included angles between polarizers and analyzers are $\pm 45^{\circ}, \mp 45^{\circ}$ respectively.

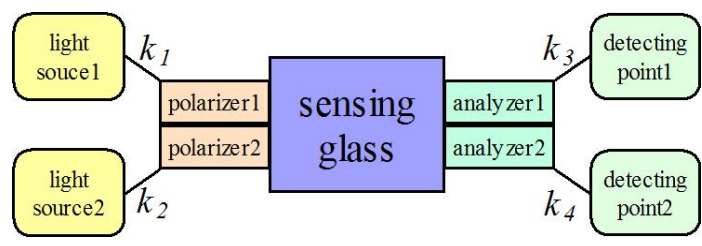

Fig. 4. Equivalent structure of sensor with difference of light path and circuit gains.

Because of the scattering of photoelectric and electronic devices, the gains of the light path and circuit channel are not absolutely equal even if the same devices are used. Assuming the attenuating coefficients from light sources to polarizers are $k_{1}, k_{2}$ and from analyzers to detecting points are $k_{3}, k_{4}$ respectively. When light source $1 I_{1}$ is lighted, the two channels of output light power are,

$$
I_{11}=k_{1} k_{3} I_{1}(1+2 \varphi), I_{12}=k_{1} k_{4} I_{1}(1-2 \varphi) .
$$

Similarly, when light source $I_{2}$ is lighted:

$$
I_{21}=k_{2} k_{3} I_{2}(1-2 \varphi), I_{22}=k_{2} k_{4} I_{2}(1+2 \varphi) .
$$

Calculate the four outputs as following:

$$
S^{\prime}=\frac{\sqrt{I_{11} \cdot I_{22}}-\sqrt{I_{12} \cdot I_{21}}}{\sqrt{I_{11} \cdot I_{22}}+\sqrt{I_{12} \cdot I_{21}}}=\frac{\sqrt{k_{1} k_{2} k_{3} k_{4} I_{1} I_{2}} \cdot 4 \varphi}{\sqrt{k_{1} k_{2} k_{3} k_{4} I_{1} I_{2}} \cdot 2}=2 \varphi .
$$

The Faraday rotation angle is obtained. Then the final compensated result of the MOCS can be achieved from Eq. 6 . 
No matter which structure of the sensing arms, the influences of light source intensity fluctuation can be eliminated from Eq. 8 or Eq. 11.

\subsection{Implementation}

The newly designed MOCS has two main modules located at the low- voltage side and high-voltage side respectively, similar to an ordinary one. The differences between them are the number of light sources or LEDs, the driving circuit with switch control, and the way of signal processing. Two extra LEDs have to be configured to arm 2 for the dual-input purpose, as shown in Fig. 5. LED1 and LED2 are alternatively lighted by a midget relay controlled by a CPU processor. The control signal has a fixed time lead relative to the sampling clock to avoid light crosstalk. The modulated light intensity is converted to an electrical voltage signal through an $\mathrm{O} / \mathrm{E}$ converter and subsequently digitalized by a multi-channel data acquisition card. The processor then splits the two outputs, $I_{1}, I_{2}$ of arm2, into four, $I_{11}, I_{12}, I_{21}, I_{22}$ through a reorganization of interval, to demodulate the DC Faraday rotation $\varphi_{N}$. The $\varphi_{x}$ demodulation of arm1 is conventional, as described in Eq. 7-Eq. 8. Three LEDs are of the same type, such as HFBR-1414, made by Agilent Co., and the correspondent O/E converter type is HFBR2414.

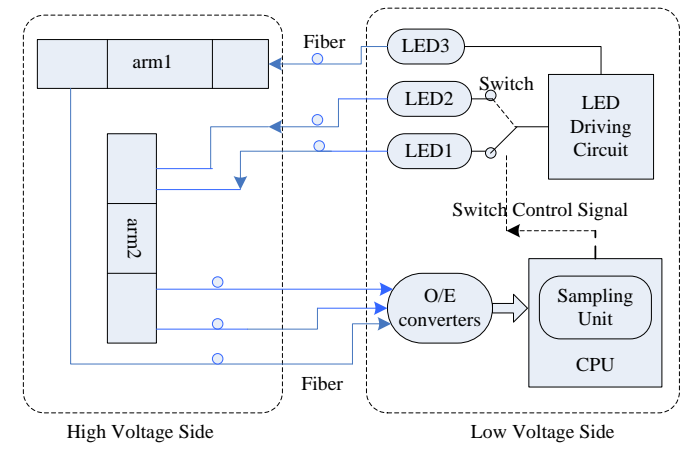

Fig. 5. Implementation of the newly-designed MOCS.

\section{Test}

The chosen sensing material of MOCS is paramagnetic glass TG20 made by SIOM, Shanghai China. Its Verdet constant temperature coefficient is about $0.3 \% /{ }^{\circ} \mathrm{C}$. The comparison tests are performed between the newly designed MOCS and an ordinary one without any compensation. They are all put in the same box whose temperature can be varied to mimic the outdoor environment. The temperature gradient is greater than $6^{\circ} \mathrm{C} / \mathrm{h}$, set according to IEC60044-8. The measurement currents are compared with a standard high- accuracy (class 0.01) current transformer in an error processor. The relative errors are plotted in Fig. 6.

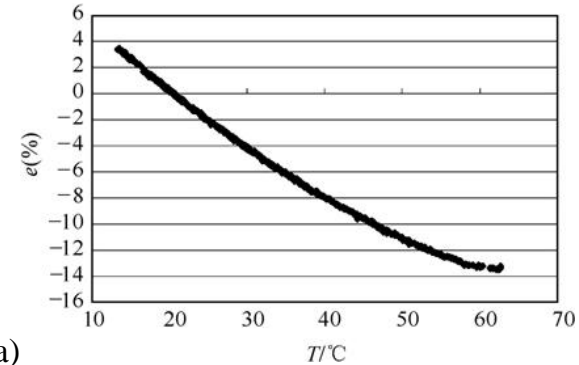

a)

Fig. 6. Temperature performance test comparison between uncompensated and compensated MOCS. a) error-T test curve of uncompensated MOCS, b) error-T test curve of compensated MOCS.

When the temperature change is about $50^{\circ} \mathrm{C}$, the error change of MOCS without any 
compensation is up to about $16 \%$, while for the newly-designed MOCS, the error change is only about $0.9 \%$ in the same temperature range. The temperature stability is increased greatly.

\section{Discussion and conclusions}

The critical factors that cause the poor temperature stability of a traditional MOCS are the Verdet constant and linear birefringence. In order to improve the temperature stability of an ordinary MOCS, a new compensation scheme based on comparative measurement has been demonstrated. The new scheme compensates the two critical factors simultaneously by introducing a reference magnetic field and by comparing the primary current magnetic field with the reference. The advantage of this method is that it is capable of performing real-time and automatic compensation without any temperature monitoring unit nor prior knowledge of the temperature characteristics of the sensing material.

A novel dual-input and dual-output sensor structure is designed for demodulating purpose of the DC Faraday rotation angle, caused by the introduced permanent magnet. In fact no matter AC or DC, Faraday rotation can be precisely demodulated in this way, furthermore the sensitivity variation caused by the scattering of light path attenuation coefficients and circuit channels' gains can be eliminated. The temperature experiment shows the validation of this compensation scheme.

However, the introduction of a reference sensing arm makes the structure a little more complicated. More light sources and fibers are necessary. The samarium cobalt magnet is brittle and has to be fixed carefully. This implies some potential instability of the MOCS. Some novel solid material is expected to provide a reliable and high magnetic field and a more elaborate structure design is required.

\section{Acknowledgment}

This research is subsidized by the National Natural Science Foundation of China (NSFC) under Grant No. 50377011. The authors would like to thank for the support.

\section{References}

[1] Menke, P., Bosselmann, T. (1995). Temperature compensation in magnetooptic AC current sensor using an intelligent AC-DC signal evaluation. J. Lightwave Technol., 13(77), 1362-1370.

[2] Wang, Z., Li, Q., Qi, Y., Huang, Z., Shi, J. (2004). Joint effect of dispersions of reflection-induced retardance and Verdet constant upon the sensitivity of an magneto-optical current sensor. Optics and Laser Technology, 36(3), 233-237.

[3] Yuri, S., Didosyan, H.H., Haberl, F. (2001). Magneto-magneto-optical current transformer of high bandwidth and large temperature range. Sensors and Actuators A: Physical, 92(1-3), 67-73.

[4] Ma, X., Luo, C. (1998). A method to eliminate birefringence of a magneto-optic AC current transducer with glass ring sensor head. IEEE Trans. Power Delivery, 13(4), 1015-1019.

[5] Iain Madden, W., Craig Michie, W., Cruden, A., Niewczas, P., McDonald, J.R. (1999). Temperature compensation for optical current sensors. Opt. Eng., 38(10), 1701-1707.

[6] Perciante, C.D., Ferrari, J.A. (2005). Faraday current sensor with temperature monitoring. Appl. Opt., 44(32), 6910-6912.

[7] International standard. (2002). IEC60044-8: Instrument transformer-Part 8: Electronic current transformer. International Electrotechnical Commission.

[8] Williams, P.A., Rose, A.H., Day, G.W., Milner, T.E., Deeter, M.N. (1991). Temperature dependence of the Verdet Constant in Several diamagnetic glass. Appl. Opt., 30(10), 1176-1178.

[9] Yi, B., Chu, B.C.B., Chiang, K.S., Chung, H.S.H. (2000). New design of optical electric-current sensor for sensitivity improvement. IEEE Trans. Instrum. Meas., 49(2), 418-423. 